International Journal of Modern Physics E

(C) World Scientific Publishing Company

\title{
PARTIAL CONSERVATION OF SENIORITY IN NUCLEI
}

\author{
P. VAN ISACKER \\ Grand Accélérateur National d'Ions Lourds, CEA/DSM-CNRS/IN2P3 \\ B.P. 55027, F-14076 Caen Cedex 5, France \\ isacker@ganil.fr \\ Received (received date) \\ Revised (revised date)
}

\begin{abstract}
A review is given of the relation between pairing, quasi-spin algebras and seniority. The former two concepts are closely connected, the relation being that the quasi-spin formalism allows an efficient solution of the pairing problem. Seniority is of much wider applicability than either pairing or quasi-spin and applications can even be extended to encompass the notion of partial symmetry. A recent application of partial conservation of seniority and its importance for nuclear isomers is discussed.
\end{abstract}

\section{Pairing, seniority and quasi-spin algebras}

The pairing interaction is a reasonable first-order approximation to the strong force between identical nucleons. For nucleons in a single- $j$ shell, as will be assumed throughout in this contribution, pairing is defined by the matrix elements

$$
\nu_{J} \equiv\left\langle j^{2} ; J M_{J}|\hat{V}| j^{2} ; J M_{J}\right\rangle=-g(2 j+1) \delta_{J 0},
$$

where $j$ is the total (orbital+spin) angular momentum of a single nucleon (hence $j$ is half-odd-integer), $J$ results from the coupling of two $j$ s and $M_{J}$ is the projection of $J$ on the $z$ axis. Furthermore, $g$ is the strength of the interaction which is attractive in nuclei $(g>0)$.

The pairing interaction was introduced by Racah for the classification of $n$ electrons in an atom 1 . He was able to derive a closed formula for the interaction energy among the $n$ electrons and to prove that any eigenstate of the pairing interaction is characterized by a 'seniority number' $v$ which corresponds to the number of electrons that are not in pairs coupled to orbital angular momentum $L=0$. Racah's original definition of seniority made use of coefficients of fractional parentage. He later noted that simplifications arose through the use of group theory 2 . Seniority turned out to be a label associated with the (unitary) symplectic algebra $\mathrm{Sp}(2 j+1)$ in the classification

$\begin{array}{ccc}\mathrm{U}(2 j+1) & \supset \mathrm{Sp}(2 j+1) & \supset \mathrm{SU}(2) \\ \downarrow & \downarrow & \downarrow \\ {\left[1^{n}\right]} & {\left[1^{v}\right]} & J\end{array}$.


Since the nucleons are identical, all states of the $j^{n}$ configuration belong to the totally antisymmetric irreducible representation (IR) $\left[1^{n}\right]$ of $\mathrm{U}(2 j+1)$. The IRs of $\operatorname{Sp}(2 j+1)$ therefore must also be totally antisymmetric of the type [ $\left.1^{v}\right]$ with allowed values of seniority $v=n, n-2, \ldots, 1$ or 0 .

In the definition (2), seniority appears as a label associated with the algebra $\operatorname{Sp}(2 j+1)$. This has the drawback that, depending on $j$, the algebra can be quite large. Matters become even more complicated when the fermions are non-identical and are assigned an intrinsic label $t$ with $2 t+1$ substates, such as is the case with nucleons which have isospin $t=\frac{1}{2}$. The total number of single-particle states is then $\Omega \equiv(2 j+1)(2 t+1)$ and the classification (2) is generalized to $\underline{3.4}$

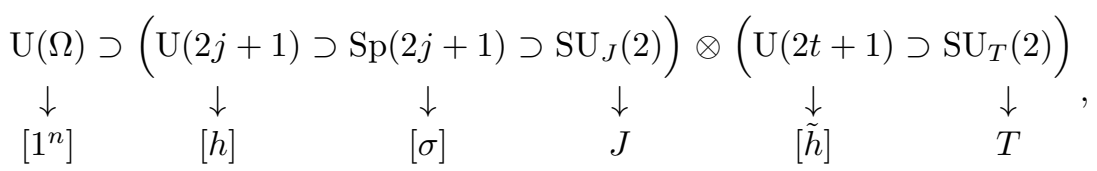

where $[h],[\tilde{h}]$ and $[\sigma]$ are Young tableaux associated with $\mathrm{U}(2 j+1), \mathrm{U}(2 t+1)$ and $\operatorname{Sp}(2 j+1)$, respectively, $J$ is the total angular momentum and $T$ the total isospin (or, in general, the total coupling of all ts). The classification (3) assumes a symmetry $\mathrm{SU}_{J}(2) \otimes \mathrm{SU}_{T}(2)$, that is, an interaction which conserves angular momentum $J$ and isospin $T$. To ensure overall antisymmetry under $\mathrm{U}(\Omega)$, the Young tableaux of $\mathrm{U}(2 j+1)$ and $\mathrm{U}(2 t+1)$ must be conjugate, that is, $[\tilde{h}]$ is obtained from $[h]$ by interchanging rows and columns. This conjugate relationship can be expressed as follows:

$$
[h]=[\overbrace{2 t+1,2 t+1, \ldots, 2 t+1}^{\tilde{h}_{2 t+1}}, \overbrace{2 t, 2 t, \ldots, 2 t}^{\tilde{h}_{2 t}-\tilde{h}_{2 t+1}}, \ldots, \overbrace{1,1, \ldots, 1}^{\tilde{h}_{1}-\tilde{h}_{2}}]
$$

and, since $[h]$ contains at most $2 j+1$ labels, this proves that overall antisymmetry requires $\tilde{h}_{1} \leq 2 j+1$. The converse relation reads

$$
[\tilde{h}]=[\overbrace{2 j+1,2 j+1, \ldots, 2 j+1}^{h_{2 j+1}}, \overbrace{2 j, 2 j, \ldots, 2 j}^{h_{2 j}-h_{2 j+1}}, \ldots, \overbrace{1,1, \ldots, 1}^{h_{1}-h_{2}}],
$$

and shows that $h_{1} \leq 2 t+1$. The requirement of overall antisymmetry sets limits on the allowed tableaux $[h]$ or $[\tilde{h}]$. Assuming for the sake of definiteness that $t \leq j$, the Young tableau of $\mathrm{U}(2 j+1)$ can be specified by $2 t+1$ labels $\tilde{h}_{i}$ instead of the $2 j+1$ normally needed. As a consequence of the form (4) of $[h]$, all non-zero labels in $[\sigma]$ must be $2 t+1,2 t, \ldots$ or 1 , and the Young tableau of $\mathrm{Sp}(2 j+1)$ must therefore be of the form

$$
[\sigma]=[\overbrace{2 t+1,2 t+1, \ldots, 2 t+1}^{\sigma_{2 t+1}}, \overbrace{2 t, 2 t, \ldots, 2 t}^{\sigma_{2 t}}, \ldots, \overbrace{1,1, \ldots, 1}^{\sigma_{1}}] .
$$

The IR of $\operatorname{Sp}(2 j+1)$ is thus characterized by $2 t+1$ labels at most. For identical nucleons, $t=0$, there is one label, namely seniority $v$. For non-identical nucleons, $t=\frac{1}{2}$, there are two such labels, introduced by Flowers 4 , which correspond to seniority $v$ and to reduced isospin $t_{v}$. The former has the same interpretation as 
in the like-nucleon case while the latter corresponds to the isospin of the nucleons which are not in pairs coupled to $J=0$.

It is clear that one quickly runs into formidable group-theoretical reduction problems if one follows the formulation associated with the classification (3). Fortunately, an alternative and simpler definition of seniority can be given in terms of algebras that do not change with $j$. The idea was simultaneously and independently proposed by Kerman $\sqrt{5}$ for $t=0$ (i.e., for identical particles) and by Helmers 6 for general $t$. The starting point is the introduction of the operators

$$
\begin{aligned}
& \hat{S}_{+}^{j}\left(m_{t}, m_{t}^{\prime}\right)=\sqrt{2 j+1}\left(a_{j, t m_{t}}^{\dagger} \times a_{j, t m_{t}^{\prime}}^{\dagger}\right)_{0}^{(0)} \equiv \hat{S}_{+}^{j}\left(m_{t}^{\prime}, m_{t}\right), \\
& \hat{S}_{-}^{j}\left(m_{t}, m_{t}^{\prime}\right)=\left(\hat{S}_{+}^{j}\left(m_{t}, m_{t}^{\prime}\right)\right)^{\dagger} \equiv \hat{S}_{-}^{j}\left(m_{t}^{\prime}, m_{t}\right), \\
& \hat{S}_{z}^{j}\left(m_{t}, m_{t}^{\prime}\right)=\sqrt{2 j+1}\left(a_{j, t m_{t}}^{\dagger} \times \tilde{a}_{j, t m_{t}^{\prime}}\right)_{0}^{(0)}-\frac{1}{2}(2 j+1) \delta_{m_{t} m_{t}^{\prime}} .
\end{aligned}
$$

The particle creation operators $a_{j m_{j}, t m_{t}}^{\dagger}$ are assigned an angular momentum $j$ with projection $m_{j}$ and an intrinsic quantum number $t$ with projection $m_{t}$. To ensure the correct transformation properties under rotations, the operators $\tilde{a}_{j m_{j}, t m_{t}} \equiv(-)^{j+m_{j}+t+m_{t}} a_{j-m_{j}, t-m_{t}}$ are introduced. Furthermore, the coupling, indicated in (7) by $\times$, refers to angular momentum and no coupling in $t$ is carried out. It can be shown 6 that the operators (7) close under commutation and generate the (unitary) symplectic algebra $\mathrm{Sp}(4 t+2)$. Furthermore, these operators are 'symplectic invariants' in the sense that they commute with all the generators of the symplectic algebra $\operatorname{Sp}(2 j+1)$ introduced in (3) . Because of this property, the IRs of $\operatorname{Sp}(2 j+1)$ [contained in the antisymmetric IR of $\mathrm{U}(\Omega)$ ] are in one-to-one correspondence with those of $\operatorname{Sp}(4 t+2)$. The two IRs are complementary, that is, one is obtained from the other by constructing the complement of the Young tableau in a rectangle with $j+\frac{1}{2}$ rows and $2 t+1$ columns and reflecting this complement along the lower-left-to-upper-right diagonal of the rectangle ${ }^{6}$. This complementary relationship between the $[\sigma] \equiv\left[\sigma_{1}, \sigma_{2}, \ldots, \sigma_{j+\frac{1}{2}}\right]$ labels of $\operatorname{Sp}(2 j+1)$ and the $[\bar{\sigma}]$ labels of $\operatorname{Sp}(4 t+2)$ can be expressed as follows:

$$
[\bar{\sigma}]=[\overbrace{j+\frac{1}{2}, j+\frac{1}{2}, \ldots, j+\frac{1}{2}}^{2 t+1-\sigma_{1}} \overbrace{j-\frac{1}{2}, j-\frac{1}{2}, \ldots, j-\frac{1}{2}}^{\sigma_{1}-\sigma_{2}} \ldots, \overbrace{1,1, \ldots, 1}^{\sigma_{j-\frac{1}{2}}-\sigma_{j+\frac{1}{2}}}] .
$$

For $t=0$ (identical fermions) the operators (7) generate the algebra $\operatorname{Sp}(2)$ which is isomorphic to $\mathrm{SU}(2)$ and which therefore has been named 'quasi-spin' algebra ${ }^{5}$. The seniority label $v$ in the classification (2) goes over into the $\mathrm{Sp}(2)$ label $(2 j-2 v+1) / 2$ which corresponds to $(2 j-2 v+1) / 4$ in $\mathrm{SU}(2)$. For $t=\frac{1}{2}$ (neutrons and protons) one finds the 'quasi-spin' algebra $\mathrm{Sp}(4) \sim \mathrm{SO}(5)$, extensively studied by Hecht 7 . The seniority and reduced isospin $\left[2^{v / 2-t_{v}}, 1^{2 t_{v}}\right]$ in the classification (3) go over in the $\mathrm{Sp}(4)$ labels $\left[\left(2 j-v+2 t_{v}+1\right) / 2,\left(2 j-v-2 t_{v}+1\right) / 2\right]$ which correspond to $\left[(2 j-v+1) / 2, t_{v}\right]$ in $\mathrm{SO}(5)$. 
The connection with pairing is established by constructing the operator

$$
\hat{Q}=\frac{1}{4} \sum_{m_{t} m_{t}^{\prime}} \hat{S}_{+}^{j}\left(m_{t}, m_{t}^{\prime}\right) \hat{S}_{-}^{j}\left(m_{t}, m_{t}^{\prime}\right),
$$

which is related to the Casimir operator of $\operatorname{Sp}(4 t+2)$ through

$$
\hat{Q}=\frac{1}{4} n(\Omega-n+2)-\frac{1}{16} \Omega(4 t+2 j+5)+\frac{1}{4} \hat{C}_{2}[\operatorname{Sp}(4 t+2)],
$$

where use is made of the complementarity relation between $\operatorname{Sp}(2 j+1)$ and $\operatorname{Sp}(4 t+2)$. Its eigenvalues are found from those of $\hat{C}_{2}[\operatorname{Sp}(4 t+2)]$ which are $\sum_{i} \bar{\sigma}_{i}\left(\bar{\sigma}_{i}+4 t+4-2 i\right)$. For $t=0, \hat{Q}$ coincides with the pairing interaction, $\hat{V}_{\mathrm{P}}=\hat{Q}$, and the following eigenvalue expression for the pairing interaction results:

$$
E_{n j}(v)=\frac{1}{4}(n-v)(2 j-n-v+3) .
$$

For $t=\frac{1}{2}$, the relation between $\hat{Q}$ and $\hat{V}_{\mathrm{P}}$ becomes

$$
\hat{V}_{\mathrm{P}}=\hat{Q}-\frac{1}{2} \hat{C}_{2}\left[\mathrm{SU}_{T}(2)\right]-\frac{1}{8} n(4 j-n),
$$

and leads to the eigenvalue expression for the isospin-invariant pairing interaction:

$$
E_{n j}\left(T, v, t_{v}\right)=\frac{1}{8}(n-v)(4 j-n-v+8)-\frac{1}{2}\left[T(T+1)-t_{v}\left(t_{v}+1\right)\right] .
$$

This succinct and simultaneous derivation of the eigenvalues in the cases of identical nucleons and of neutrons and protons, illustrates the power of the quasispin formulation of the pairing problem.

\section{Conservation of seniority}

While the preceding analysis shows that the eigenstates of a pairing interaction carry the quantum numbers associated with seniority, it does not answer the question what are the necessary and sufficient conditions for a general interaction to conserve seniority. This problem has been worked out in its full generality for identical particles (i.e., $t=0$ ). In that case, a rotationally invariant two-body interaction $\hat{V}$ is completely specified by the matrix elements $\nu_{J}$ in Eq. (1) with $J=0,2, \ldots, 2 j-1$. The necessary and sufficient conditions for the conservation of seniority can then be written as 8910

$$
\sum_{J=2}^{2 j-1}(2 J+1) \sqrt{2 I+1}\left(\frac{\delta_{J I}}{\sqrt{(2 J+1)(2 I+1)}}+2\left\{\begin{array}{lll}
j & j & J \\
j & j & I
\end{array}\right\}-\frac{4}{\left(4 j^{2}-1\right)}\right) \nu_{J}=0
$$

with $I=2,4, \ldots, 2 j-1$, and where the symbol between curly brackets is a Racah coefficient. No condition on the matrix elements $\nu_{J}$ is obtained for $j=3 / 2,5 / 2$ and $7 / 2$, one condition for $j=9 / 2,11 / 2$ and $13 / 2$, and so on. Clearly, the conditions (14) are much weaker than the requirement that the interaction be of pairing character but still many of the results of the quasi-spin formalism remain valid. 


\section{Partial conservation of seniority}

It was shown recently 11 that the seniority quantum number occasionally can be conserved for some but not all eigenstates. One may characterize this as a partial conservation of seniority, akin to the notion of partial dynamical symmetry 12 . There are a number of 'trivial' examples of partial conservation of seniority. For example, if the total angular momentum $J$ is odd, a four-particle state cannot be of seniority $v=0$ or $v=2$ and must necessarily have seniority $v=4$. Also, for $J>2 j$ the four-particle state must be of seniority $v=4$. These trivial cases are not of concern here. The discussion of partial conservation of seniority so far has been confined to the case of identical fermions which is assumed in this and the following section.

A non-trivial example of partial conservation of seniority was discovered by Escuderos and Zamick ${ }^{13}$ who pointed out that four identical fermions with $j=9 / 2$ display one $J=4$ and one $J=6$ state both of which have seniority $v=4$ for an arbitrary interaction. This is an example of a partial symmetry, where seniority is broken for most but not for all states. An analysis of the four-particle system shows 11 that the two eigenstates can be written as

$$
\begin{aligned}
& \left|(9 / 2)^{4}, v=4, J=4\right\rangle=\sqrt{\frac{25500}{25591}}\left|(9 / 2)^{4}[22] 4\right\rangle-\sqrt{\frac{91}{25591}}\left|(9 / 2)^{4}[24] 4\right\rangle, \\
& \left|(9 / 2)^{4}, v=4, J=6\right\rangle=\sqrt{\frac{27132}{27257}}\left|(9 / 2)^{4}[24] 6\right\rangle+\sqrt{\frac{125}{27257}}\left|(9 / 2)^{4}[26] 6\right\rangle .
\end{aligned}
$$

Furthermore, the two states have the eigenenergies

$$
\begin{aligned}
& E\left[(9 / 2)^{4}, v=4, J=4\right]=\frac{68}{33} \nu_{2}+\nu_{4}+\frac{13}{15} \nu_{6}+\frac{114}{55} \nu_{8}, \\
& E\left[(9 / 2)^{4}, v=4, J=6\right]=\frac{19}{11} \nu_{2}+\frac{12}{13} \nu_{4}+\nu_{6}+\frac{336}{143} \nu_{8} .
\end{aligned}
$$

The wave functions (15) are identical to those of Eq. (8) of Ref 11 but written here in a Gram-Schmidt basis. For the definition of this basis one starts, for $J=4$, from the non-orthogonal set $\left|(9 / 2)^{4}\left[I I^{\prime}\right] 4\right\rangle$ with $\left[I I^{\prime}\right]=[04]$, [22] and [24]. (The labels $I$ and $I^{\prime}$ refer to the angular momenta of the two pairs as they appear in a two-fermion-pair basis 11 .) The second and third states obtained after the GramSchmidt orthonormalization are orthogonal to $\left|(9 / 2)^{4}[04] 4\right\rangle$, and hence by definition have seniority $v=4$. A similar argument is valid for $J=6$ where the non-orthogonal set $\left|(9 / 2)^{4}\left[I I^{\prime}\right] 6\right\rangle$ has $\left[I I^{\prime}\right]=[06],[24]$ and [26]. Consequently, the states (15) have seniority $v=4$.

In summary, the wave functions of the two states (15) are pre-determined and their energies are linear combinations of the interaction matrix elements $\nu_{J}$ with coefficients that are rational non-negative numbers. These results are valid for an arbitrary interaction among $j=9 / 2$ fermions. According to Talmi's definition 14 , the states are therefore solvable, independent of whether the interaction conserves seniority or not. Although a mathematical derivation can be given of the necessary conditions for the existence of such solvable states, a simple, intuitive reason for 
it is still lacking. Some progress towards this goal has been made 15 , and a partial understanding of the coefficients entering the energy expressions (16) has been achieved.

The property of partial conservation of seniority may appear rather academic because it only occurs four identical fermions in a single shell with $j=9 / 2$. Nevertheless, as argued in the next section, this peculiar exception is crucial for the existence of seniority isomers in nuclei.

\section{Seniority isomers in semi-magic nuclei}

In nuclei, states with low seniority generally occur at low energy. For example, the ground state of an even-even semi-magic nucleus has approximately $v \approx 0$ (all nucleons in pairs coupled to $J=0$ ) while its lowest levels with angular momenta $J=$ $2,4,6, \ldots$ usually have $v \approx 2$ (containing one 'broken' pair with $J \neq 0$ ). Seniority isomerism is expected to occur in semi-magic nuclei because electric quadrupole (E2) transitions between $v=2$ states are hindered, especially when the valence shell is close to half-filled. This result is a consequence of the fact that the matrix elements of the quadrupole operator between states with seniority $v=2$ vanish at mid shell 8 .

Examples of seniority isomers have been found in the $N=50$ isotones with protons dominantly confined to the $1 g_{9 / 2}$ shell. In particular, the $J^{\pi}=8^{+}$levels in ${ }^{94} \mathrm{Ru}(Z=44)$ and ${ }^{96} \mathrm{Pd}(Z=46)$ have half-lives of 71 and $2.2 \mu$ s, respectively, resulting from a combination of slow E2 decay and a small energy difference with

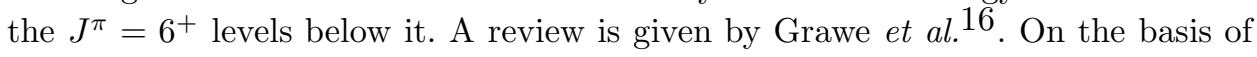
similar arguments one would expect the same phenomenon to occur in the neutronrich nickel $(Z=28)$ isotopes ${ }^{72} \mathrm{Ni}$ and ${ }^{74} \mathrm{Ni}$ with neutrons dominantly confined to the $1 g_{9 / 2}$ shell but this does not seem to be the case 17 .

The seniority classification is a good approximation for $n$ identical nucleons in a $j=9 / 2$ shell. This is a trivial statement if only one state exists for a given particle number $n$ and angular momentum $J$, in which case seniority must be exact. If two states with different seniority and the same $J$ occur, it can be shown that for any reasonable nuclear interaction $\hat{V}$ the off-diagonal matrix element is small compared to the states' energy difference. The proof that seniority mixing is negligible for the $J^{\pi}=4^{+}$and $6^{+}$states of a $(9 / 2)^{4}$ system is more subtle and is crucial for the existence of seniority isomers. There are three states for each of these angular momenta, two of which, with $v=2$ and $v=4$, are close in energy and could possibly strongly mix. However, the $v=4$ members of these closely-spaced doublets are the $J^{\pi}=4^{+}$and $6^{+}$states discussed in Sect. 3 which have exact seniority $v=4$ for any interaction. As a result, breaking of seniority only arises through the mixing between the $v=2$ and the higher-lying $v=4$ states and, by the same argument as above, this mixing is found to be small.

In Fig. 1 the results of a calculation for the $N=50$ even-even isotones are compared to the observed spectra ${ }^{18}$. The two-particle and the two-hole spectra, 


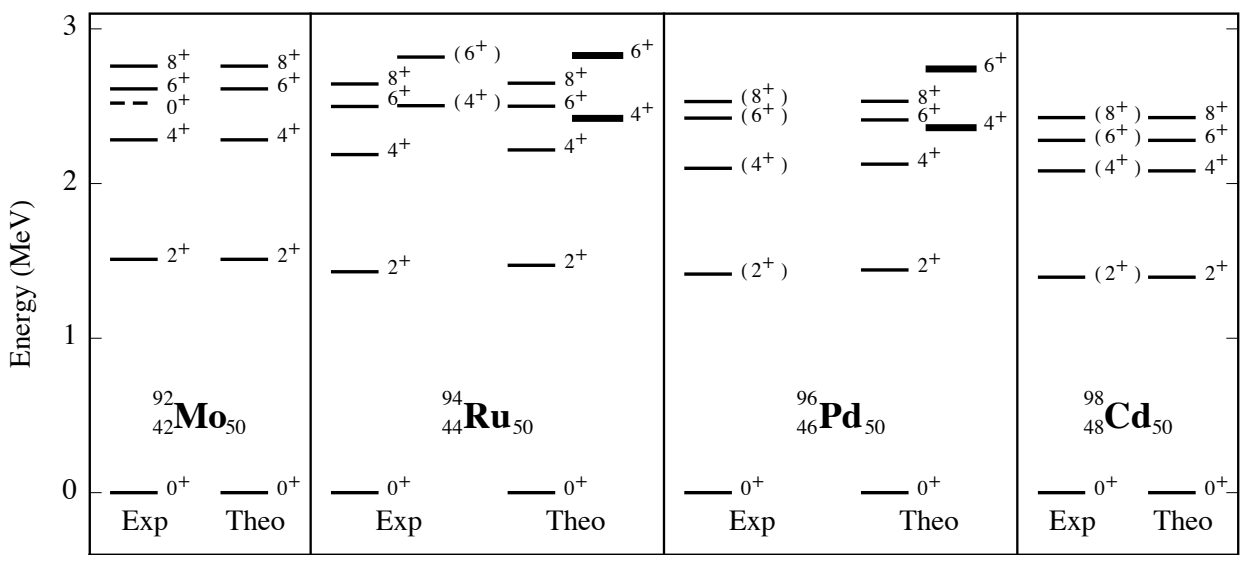

Fig. 1. The low-energy spectra of the $N=50$ isotones ${ }^{92} \mathrm{Mo},{ }^{94} \mathrm{Ru},{ }^{96} \mathrm{Pd}$ and ${ }^{98} \mathrm{Cd}$. The left-hand parts of each panel show the observed levels while the right-hand parts contain the results of a $\left(1 g_{9 / 2}\right)^{n}$ shell-model calculation with linearly varying two-body matrix elements. The $J^{\pi}=4^{+}$ and $6^{+}$levels with exact $v=4$ are shown with thick lines; the dashed line corresponds to an intruder level.

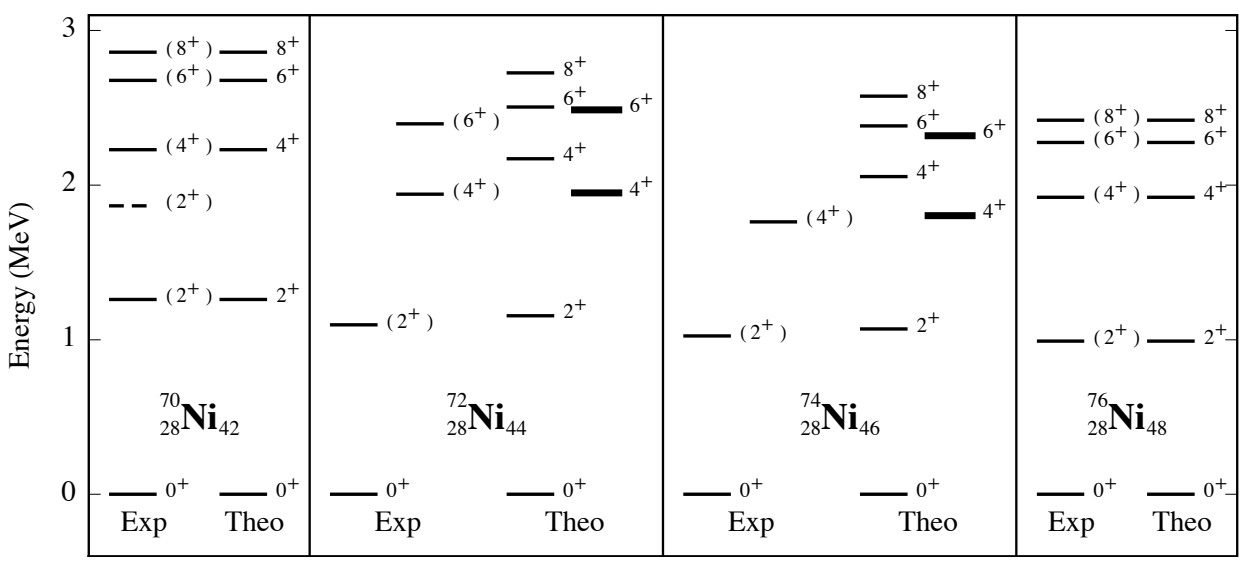

Fig. 2. The low-energy spectra of the nickel isotopes ${ }^{70-76} \mathrm{Ni}$. See caption of Fig. 1

${ }^{92} \mathrm{Mo}$ and ${ }^{98} \mathrm{Cd}$, determine the two-body matrix elements $\nu_{J}$. Different procedures can be followed to fix these matrix elements, all leading to similar results, and those shown in Fig. 1 are obtained by varying $\nu_{J}$ linearly between ${ }^{92} \mathrm{Mo}$ and ${ }^{98} \mathrm{Cd}$. The description of the two intermediate isotopes, ${ }^{94} \mathrm{Ru}$ and ${ }^{96} \mathrm{Pd}$, should then be rather accurate, albeit very phenomenological. In ${ }^{94} \mathrm{Ru}\left(4_{2}^{+}\right)$and $\left(6_{2}^{+}\right)$levels are observed at energies of 2503 and $2818 \mathrm{keV}$, respectively 19 ; these are possible candidates for the states with exact $v=4$ (thick lines) which are calculated at 2422 and $2828 \mathrm{keV}$. In Fig. 2 are shown the observed spectra of the nickel isotopes 18 , compared to a calculation similar to the one performed for the $N=50$ isotones. 
There is a striking difference between the calculated four-particle and four-hole spectra of the $N=50$ isotones and those of the nickel isotopes: the $v=4, J^{\pi}=4^{+}$ and $6{ }^{+}$states are yrast in ${ }^{72} \mathrm{Ni}$ and ${ }^{74} \mathrm{Ni}$ but not in ${ }^{94} \mathrm{Ru}$ and ${ }^{96} \mathrm{Pd}$. This drastically changes the E2-decay pattern. The 'typical' decay with small $B(\mathrm{E} 2)$ values between $v \approx 2$ states is characteristic of the seniority classification in nuclei near mid-shell $\left(n \approx j+\frac{1}{2}\right)$ and is at the basis of the explanation of seniority isomers 16 . This situation applies to ${ }^{94} \mathrm{Ru}$ and ${ }^{96} \mathrm{Pd}$ where the $v \approx 2$ states are yrast. On the other hand, the E2 decay pattern, as it is calculated in ${ }^{72} \mathrm{Ni}$ and ${ }^{74} \mathrm{Ni}$ where the yrast $J^{\pi}=4^{+}$and $6^{+}$levels have $v=4$, leads to fast E2 decay in the yrast band. As a consequence, the $J^{\pi}=8^{+}$and $6^{+}$levels are unlikely to be isomeric in this case.

Given that approximate analytic expressions are available for the energies of all relevant states, conditions for the existence of isomers can easily be established. This shows that their existence is predominantly governed by the quadrupole pairing matrix element $\nu_{2}$ of the nucleon-nucleon interaction 20 .

\section{Summary}

In this contribution the relation between pairing, seniority and quasi-spin algebras was reviewed. It was argued that seniority is of broader applicability than pairing or quasi-spin. In a few special cases, the conservation of seniority turns out to be partial, and this has important implications for the existence of seniority isomers in nuclei.

\section{References}

1. G. Racah, Phys. Rev. 63 (1943) 367.

2. G. Racah, Phys. Rev. 76 (1949) 1352.

3. G. Racah, L. Farkas Memorial Volume, (Research council of Israel, Jerusalem, 1952), p. 294.

4. B.H. Flowers, Proc. Roy. Soc. (London) A 212 (1952) 248.

5. A.K. Kerman, Ann. Phys. (NY) 12 (1961) 300.

6. K. Helmers, Nucl. Phys. 23 (1961) 594.

7. K.T. Hecht, Phys. Rev. 139, B794 (1965); Nucl. Phys. A 102, 11 (1967); Nucl. Phys. A 493, 29 (1989).

8. I. Talmi, Simple Models of Complex Nuclei, (Harwood, Chur, 1993).

9. D.J. Rowe and G. Rosensteel, Phys. Rev. Lett. 87 (2001) 172501.

10. G. Rosensteel and D.J. Rowe, Phys. Rev. C 67 (2003) 014303.

11. P. Van Isacker and S. Heinze, Phys. Rev. Lett. 100 (2008) 052501.

12. Y. Alhassid and A. Leviatan, J. Phys. A 25 (1992) L1285.

13. A. Escuderos and L. Zamick, Phys. Rev. C 73 (2006) 044302.

14. I. Talmi, Nucl. Phys. A 846 (2010) 31.

15. L. Zamick and P. Van Isacker, Phys. Rev. C 78 (2008) 044327.

16. H. Grawe et al., Prog. Part. Nucl. Phys. 38, 15 (1997).

17. M. Sawicka et al., Phys. Rev. C 68, 044304 (2003).

18. http://www.nndc.bnl.gov, National Nuclear Data Center.

19. W.J. Mills et al., Phys. Rev. C 75, 047302 (2007).

20. P. Van Isacker, to be published. 